\title{
Recurrence of an aggressive sacral hemangioma with atypical radiological features after surgical resection: A case report and literature review
}

\author{
Guan-Xi Wang \\ Yun-Qing Chen \\ Affiliated Hospital of Qingdao University \\ Yan Wang \\ Affiliated Hospital of Qingdao University \\ Bin wang \\ Affiliated Hospital of Qingdao University \\ Rui-Zhi Zhou \\ Affiliated Hospital of Qingdao University \\ Chuan-Ping Gao ( $\nabla$ gaochuanping2021@163.com ) \\ Affiliated Hospital of Qingdao University
}

Case Report

Keywords: vertebral hemangioma, aggressive, computed tomography, magnetic resonance imaging

Posted Date: February 21 st, 2022

DOI: https://doi.org/10.21203/rs.3.rs-1374878/v1

License: (9) This work is licensed under a Creative Commons Attribution 4.0 International License. Read Full License 


\section{Abstract}

Background: Aggressive vertebral hemangiomas (VHs) are uncommon in adults. Although most have typical radiographic features, some present with atypical findings, which makes their diagnosis difficult. Aggressive VHs have a high likelihood of recurrence even after resection.

Case presentation: A 52-year-old woman presented with a 3-month history of right lower extremity pain and numbness. On physical examination, the sacrum was tender, her mobility was limited, and right plantar flexion strength was $4 / 5$ on manual muscle testing. Computed tomography (CT) revealed osteolytic bony destruction from S1 to S2. Magnetic resonance imaging (MRI) showed a sacral mass invading the S1 vertebral endplate and compressing the dural sac; the mass was heterogeneously hypointense on T1-weighted imaging, hyperintense on T2-weighted imaging, and heterogeneously enhanced. Six months after surgical resection of the mass, the patient presented with back pain and bilateral extremity numbness. MRI showed lesion recurrence with thecal sac compression.

Conclusions: Aggressive VHs may exhibit atypical radiological findings that include osteolytic vertebral bony destruction, spinal canal extension, heterogeneous signal intensity on T1- and T2-weighted imaging, and heterogeneous enhancement. These can make accurate preoperative diagnosis difficult; biopsy may be necessary. Surgical decompression and resection are recommended for aggressive VHs. Nonetheless, recurrence may still occur despite resection.

\section{Background}

Vertebral hemangiomas (VHs) are one of the most common benign spinal vascular malformations. The diagnosis is based on their appearance on computed tomography (CT) and magnetic resonance imaging (MRI). Typical hemangiomas are characterized by coarsened vertical trabeculae on plain radiography and $\mathrm{CT}$; they are hyperintense on both T1- and T2-weighted MRI. Aggressive VHs may exhibit vertebral osteolytic bony destruction, compression fractures, or other atypical features and may extend into the spinal canal and/or paravertebral space, which can cause spinal cord compression and neurological deficit [1]. We report a patient with an aggressive $\mathrm{VH}$ of the sacrum that exhibited atypical radiologic features and recurred 6 months after surgical resection.

\section{Case Presentation}

A 52-year-old woman presented with a 3-month history of intermittent numbness and pain in the right lower extremity that persisted despite conservative treatment. Her medical history was unremarkable. Physical examination revealed limited mobility and tenderness and pain to percussion over the right sacrum. Right plantar flexion strength was graded $4 / 5$ on manual muscle testing. Sensory testing was normal.

CT showed bony destruction of the upper sacrum with preservation of the sacral crest and a soft tissue mass protruding into the spinal canal (Fig. 1A, B). On MRI, the mass was located in the S1 vertebral body 
and appeared hypointense on T1-weighted imaging and heterogeneously hyperintense on T2-weighted imaging (Fig. 1C, D). Thecal sac compression, vertebral endplate disruption, and heterogenous contrast enhancement were also shown (Fig. 1D, E, F). Our preliminary diagnosis was solitary bone plasmacytoma.

Resection and segmental fixation with instrumentation was performed under general anesthesia. A $16 \mathrm{~cm}$ midline lumbosacral incision was made to expose the lumbosacral junction and L5 and S1 laminectomies were performed. The lesion was located ventral to the thecal sac at S1 and measured $5 \times$ $4 \times 6 \mathrm{~cm}$ in size. It was well demarcated, reddish in color, and tough in consistency. Complete resection was achieved without leaving any obvious residual while protecting the surrounding nerves and vessels. For fixation, screws were placed in both iliac crests and L5 pedicles and connected with rods. After hemostasis was achieved, a drain was placed and the wound was closed in layers.

Histopathologic examination of the surgical specimen showed the lesion was composed of thin-walled vessels lined with a single layer of flat endothelial cells. The vessels had different diameters and varying wall thickness and were filled with blood, cellulose, and inflammatory necrosis (Fig. 2).

After surgery, the patient reported improvement in her right lower extremity numbness and pain. Six months later, she developed lumbosacral pain that rapidly progressed. Repeat MRI showed a larger S1-2 mass than before as well as thecal sac compression (Fig. 3A, B). The patient declined another resection and elected conservative medical treatment.

\section{Discussion And Conclusions}

VHs are common benign lesions characterized by proliferating blood vessels. They are considered dysemryogenic or hamartomatous in origin. Perman first described the radiographic features of typical VHs in 1926 [2]. The prevalence of $\mathrm{VH}$ is approximately $11 \%$ in adults [3]. The prevalence of hemangiomas seems to increase with age.

The vast majority of VHs are asymptomatic and quiescent. They are usually found incidentally on MRI or CT. VHs are most frequent in the thoracic spine, followed by the lumbar and cervical spine; sacral involvement is rare [1]. Multilevel involvement occurs in up to $30 \%$ of cases. Although they are usually confined to the vertebral body, extension into the posterior elements may occur.

Plain radiography may reveal either parallel linear streaks or a "honeycomb" appearance. CT can also demonstrate the classic "honeycomb" sign or vertically oriented vertebral lucencies separated by thickened trabecular bone. Typical VHs with predominant fat appear as well-defined hyperintense lesions on T1- and T2-weighted imaging.

VHs can grow quickly to extend beyond the vertebral cortex into the paravertebral and/or epidural space where they may compress the spinal cord and/or nerve roots. These lesions are called aggressive $\mathrm{VHs}$ [4] and may present with myelopathy, cauda equina syndrome, or radiculopathy. The usual presenting 
symptoms are lower extremity numbness associated with progressive motor weakness; sphincteric disturbance occurs late $[5,6]$.

Atypical VHs have less fat but higher vascular content than typical ones [4]. In 2019, Wang et al. [1] summarized imaging characteristics of aggressive VHs and described the following as atypical features: (1) expansive and osteolytic vertebral bony destruction; (2) vertebral compression fractures; (3) multilevel involvement or longitudinal soft tissue extension greater than one segment; (4) epidural soft tissue compressing the spinal cord; (4) predominant involvement of the lamina and/or pedicle; and (5) atypical MRI signals. In their study, $36 \%$ of aggressive VHs exhibited atypical findings; only one lesion was located in the sacrum. Their review of the literature over the previous 20 years revealed 45 atypical aggressive VHs.

Our patient had an aggressive $\mathrm{VH}$ that originated in the sacrum and exhibited atypical features. This lesion showed osteolytic bony destruction and did not have a "honeycomb" appearance or "polka-dot sign." It was isointense on T1-weighted imaging, hyperintense on T2-weighted imaging, and heterogeneously enhanced. The superior vertebral endplate of S1 was clearly interrupted on sagittal MRI. The lesion occupied all of S1 and involved the posterior aspect of S2. Epidural osseous compression caused symptoms of radiculopathy. Therefore, 5 radiological atypical features were present.

Decompression with laminectomy should be performed in patients with rapid and progressive neurological deficit. Reported cure rates of laminectomy for VHs without extraosseous soft tissue extension range from $70-80 \%[7,8]$. Frank et al. [9] reported six VH patients who underwent decompressive laminectomy; among these, two experienced recurrence and required reoperation. For aggressive $\mathrm{VHs}$ that cause cord compression and neurological deficit, more radical surgical resection has been advocated. The reported rates of recurrence after resection of aggressive VHs vary between studies. Goldstein et al. [10] reported two recurrences in 68 patients; the time to recurrence after surgery was 4.4 and 5.3 years, respectively. In a retrospective review of 20 patients treated for aggressive VHs, three had undergone surgical decompression at other institutions [11]. Therefore, recurrence of aggressive VHs after resection depends on not only lesion extension but also the experience of the operator. Our patient experienced recurrence 6 months after surgery and presented with worsening lower back pain. MRI showed an increase in soft tissue mass size and severe thecal sac compression.

According to the aggressive $\mathrm{VHs}$ with atypical radiological features are uncommon, accurate diagnosis before operation is challenge for radiologist. The lesions with sclerotic margin can differentiated from the most majority of malignance bone tumours. Aggressive VHs grow slowly because of the nature. Furthermore, familiar with atypical radiological features of aggressive VHs summarized by Wang et al [1] may provide clues for diagnosis. Biopsy is inevitable for some patients which may not make the final diagnosis and only revealed necrosis tissue and bone tissue.

The differential diagnosis of $\mathrm{VH}$ includes metastasis, solitary plasmacytoma, and epithelioid hemangioendothelioma. Osteolytic vertebral metastases may present with cortical expansion and associated soft tissue mass. Definitive diagnosis can be difficult and often requires multiple imaging 
modalities in addition to histopathological examination [12]. The classic radiological appearance of solitary bone plasmocytoma has been described as a "mini brain" appearance on axial CT and MRI. However, one-third of cases may mimic aggressive VH, which makes accurate diagnosis challenging [12]. Epithelioid hemangioendothelioma is a rare mesenchymal neoplasm that shows a lytic pattern of bony destruction on CT with mixed signal intensity on T1-and T2-weighted imaging.

\section{Abbreviations}

VHs, vertebral hemangiomas; MRI, magnetic resonance imaging;STIR, short time inversion recovery; CT, computed tomography

\section{Declarations}

\section{Acknowledgements}

We thank the patient for providing consent to publish this case report and Liwen Bianji (Edanz) (https://www.liwenbianji.cn) for editing the language of a draft of this manuscript.

\section{Author contributions}

CPG conceived the idea of the case report. GCW and CPG wrote the manuscript. YW treated the patients and participated in the conception of the study. YQC interpreted the data and prepare the figures to draft the manuscript. BW and RZZ revised the manuscript. All authors read and approved the final manuscript.

\section{Funding}

Funding information is not available.

\section{Availability of data and materials}

All data concerning the case are presented in the manuscript.

\section{Ethics approval and consent to participate}

All consent procedures and details of this study were approved by the Institutional Review Board of the affiliated hospital of Qingdao University囚QYFY WZLL 26806囚.

\section{Consent for publication}

Written informed consent was obtained from the patient for the publication of this report and any accompanying images.

\section{Competing interests}

The authors of this work have no competing interests to disclose. 


\section{Author details}

${ }^{1}$ Department of Radiology, Songshan Hospital of Qingdao University Medical College University. ${ }^{2}$ Department of Pathology,the affiliated hospital of Qingdao University. ${ }^{3}$ Department of Radiology,the affiliated hospital of Qingdao University.

Corresponding author: Chuanping Gao

E-mail:gaochuanping2021@613.com

\section{References}

1. Wang B, Zhang LH, Yang SM, Han SB, Jiang L, Wei F, Yuan HS, Liu XG, Liu ZJ. Atypical Radiographic Features of Aggressive Vertebral Hemangiomas. J Bone Joint Surg Am. 2019; 101:979-86.

2. Perman E. On haemangiomata in the spinal column. Acta Chit Scand. 1926;61:91-105.

3. Huvos AG. Hemangioma, lymphangioma, angiomatosis/ lymphangiomatosis, glomus tumor. In: Huvos AG, editor. Bone tumors: diagnosis, treatment, and prognosis. 2nd ed. Philadelphia: Saunders; 1991. p. 553-78.

4. Gaudino S, Martucci M, Colantonio R, Lozupone E, Visconti E, Leone A, Colosimo C.A systematic approach to vertebral hemangioma. Skeletal Radiol. 2015; 44:25-36.

5. Dang L, Liu C, S Yang HM, Jiang L, Liu ZJ, Liu XG,Hui Shu Yuan, Wei F, Yu M.Aggressive vertebral hemangioma of the thoracic spine without typical radiological appearance. Eur Spine J. 2012; 21:1994-9.

6. Nassar SI, Hanbali FS, Haddad MC, Fahl MH. Thoracic vertebral hemangioma with extradural extension and spinal cord compression. Case report. Clin Imaging. 1998; 22:65-68.

7. Ergun $\mathrm{T}$, Lakadamyali $\mathrm{H}$, Lakadamyali $\mathrm{H}$, Mukaddem A. Acute spinal cord compression from an extraosseous vertebral hemangioma with hemorrhagic components: a case report. J Manipulative Physiol Ther. 2007; 30:602-6.

8. Mohan V, Gupta SK, Tuli SM, Sanyal B. Symptomatic vertebral haemangiomas. Clin Radiol.1980;31: $575-9$.

9. Fox MW, Onofrio BM. The natural history and management of symptomatic and asymptomatic vertebral hemangiomas. J Neurosurg.1993; 78:36-45.

10. Acosta FL, Sanai N, Chi JH, Dowd CF, Chin C, Tihan T, Chou D, Weinstein P R, Ames CP.Comprehensive Management of Symptomatic and Aggressive Vertebral Hemangiomas. Neurosurg Clin N Am.2008:19;17-29.

11. Goldstein CL,Varga PP, Gokaslan ZL, Boriani S, Luzzati A, Rhines L, Fisher C G, Chou D, Williams RP, Dekutoski MB, Quraishi NA, Bettegowda C, Kawahara N, Fehlings MG.Spinal Hemangiomas: Results of Surgical Management for Local Recurrence and Mortality in a Multicenter Study. Spine.2015:140: $656-64$. 
12. Wang B, Meng N, Zhuang HQ, Han SB, Yang SM, Jiang L, Wei F, Liu XG, Liu ZJ.The Role of Radiotherapy and Surgery in the Management of Aggressive Vertebral Hemangioma: A Retrospective Study of 20 Patients. Med Sci Monit.2018: 24;6840-50.

13. Rodallec MH, Feydy A, Larousserie F, Anract P, Campagna R, Babinet A, Zins M, Drapé JL. Diagnostic imaging of solitary tumors of the spine: what to do and say. Radiographics. 2008;28:1019-41.

\section{Figures}
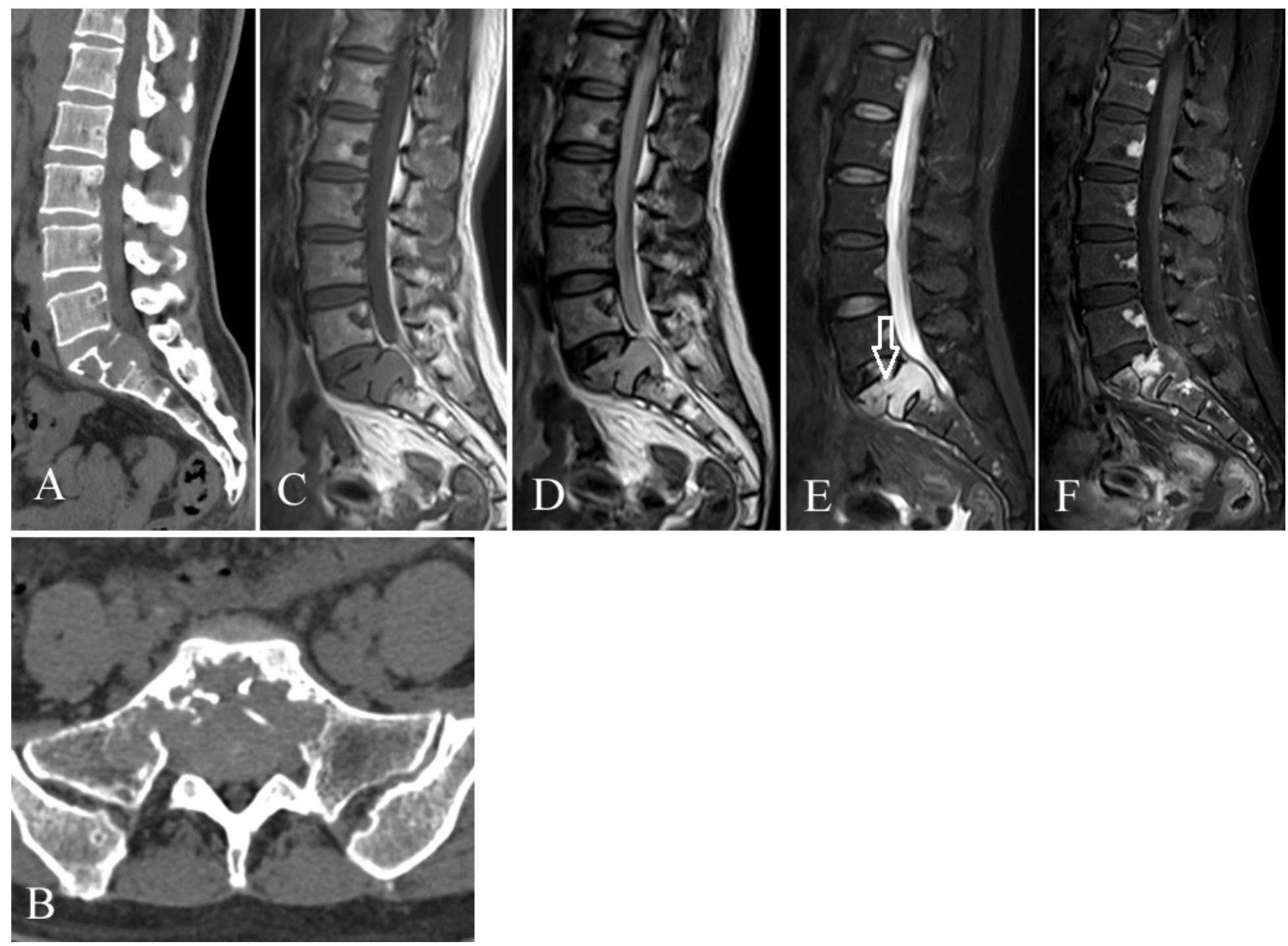

\section{Figure 1}

Sagittal (A) and axial (B) computed tomography of the lumbosacral spine showed osteolytic bony destruction at $\mathrm{S} 1$ and $\mathrm{S} 2$ with preservation of the sacral crest. On sagittal magnetic resonance imaging $(C, D, E, F)$, the lesion appeared hypointense on T1-weighted sequences and heterogeneously hyperintense on T2-weighted and short time inversion recovery sequences. The lesion exhibited heterogeneous contrast enhancement and involved the S1 endplate (arrow)and S2 vertebral body. A soft tissue mass compressed the thecal sac. 


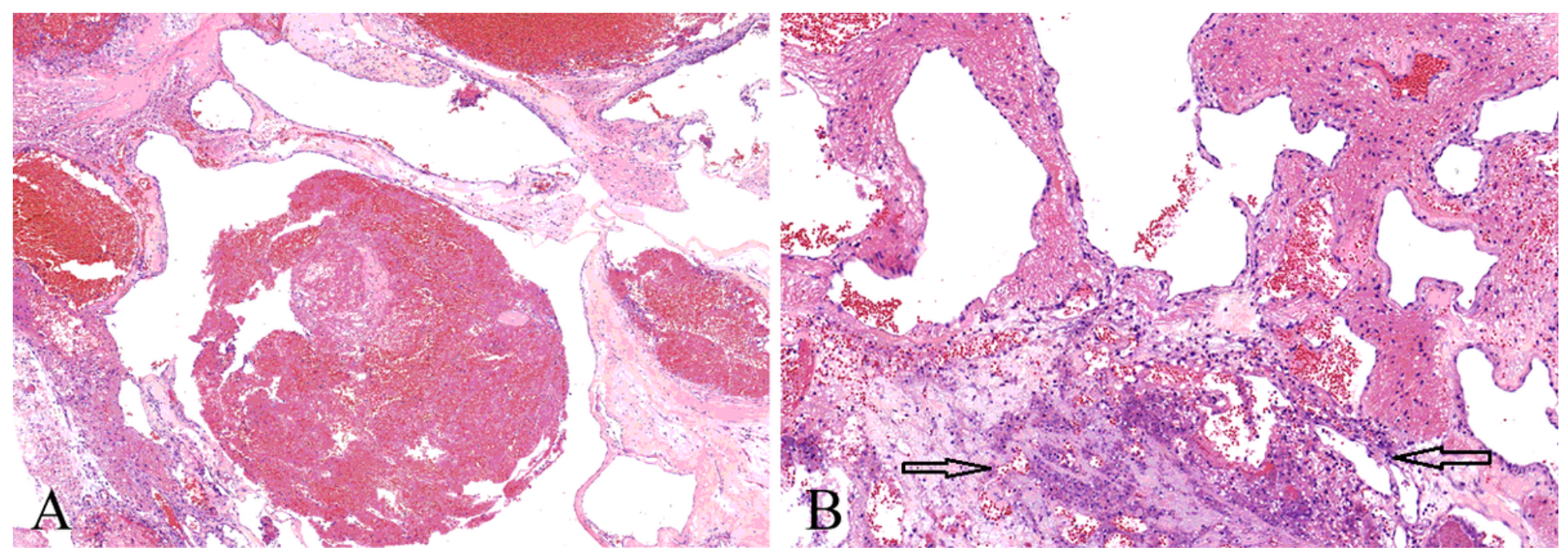

Figure 2

A photomicrograph (200x) demonstrates blood-filled channels of different diameters (A) with an area of inflammatory necrosis (B, arrow).
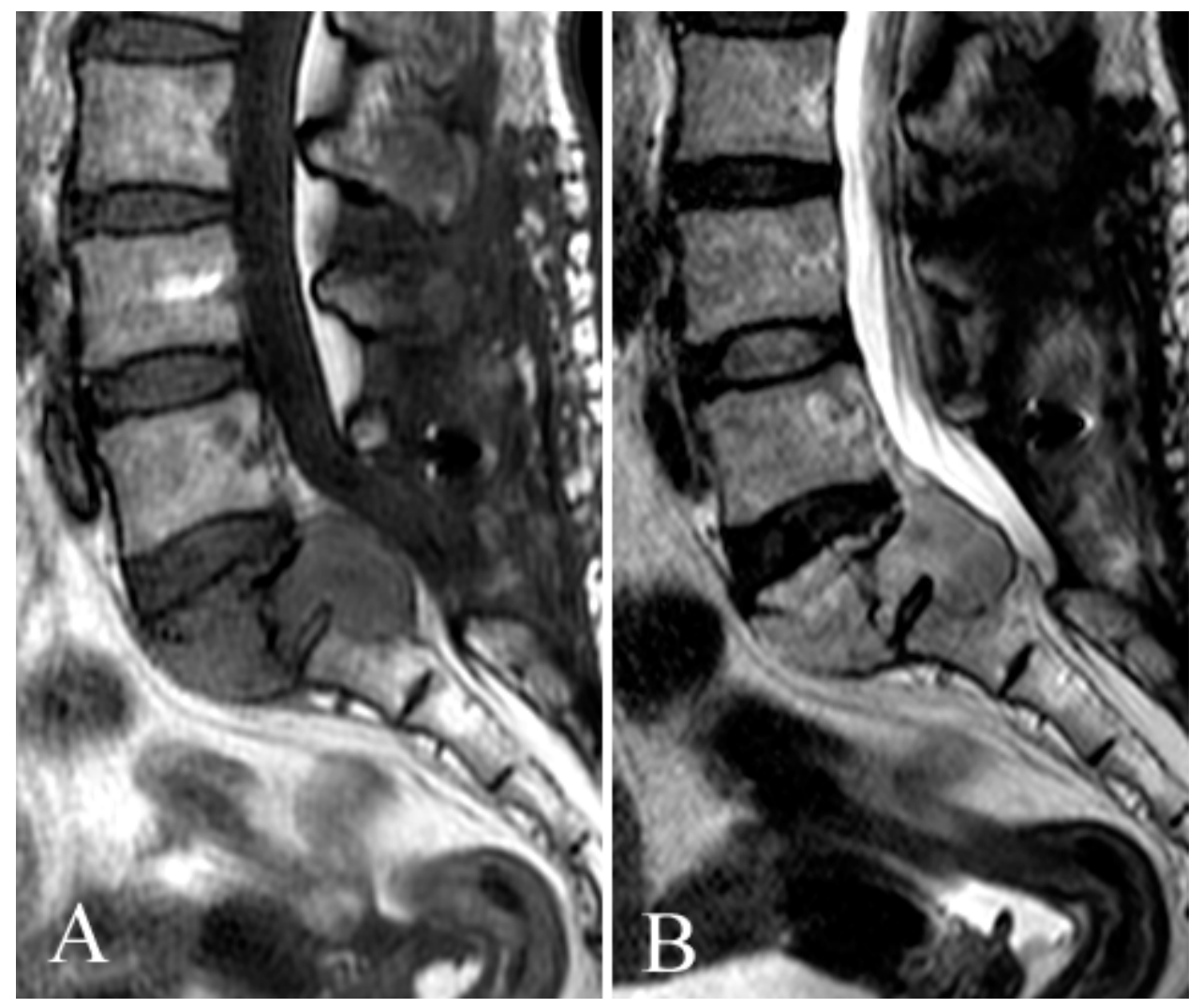

Figure 3

Sagittal magnetic resonance imaging (A, B) 6 months after surgery showed a sacral mass larger than before and thecal sac compression. 